\title{
Automatización de los procesos de secado y selección del cacao ecuatoriano conservando las características sensoriales y previniendo riesgos laborales
}

\begin{abstract}
RESUMEN
El cacao en grano es una fruta tropical considerada como un patrimonio en el Ecuador y que constituye el $60 \%$ de la calidad premium en chocolates a nivel mundial, posee características sensoriales inigualables por otros países productores. Las actividades manuales de secado y selección contienen muchos riesgos laborales, afectando la seguridad y el bienestar de las personas que se dedican a estas tareas, siendo el riesgo ergonómico de nivel intolerable y que requiere intervención inmediata. Esta investigación proporciona mecanismos automatizados seguros para las operaciones a través de metodologías validadas internacionalmente, sin afectar las características sensoriales del cacao ecuatoriano.
\end{abstract}

Palabras Clave: Características sensoriales; riesgos laborales; seguridad en maquinarias.

AUTOMATION OF THE DRYING PROCESSES AND SELECTION OF THE ECUADORIAN COCOA CONSERVING SENSORY CHARACTERISTICS AND PREVENTING LABOR RISKS

\section{ABSTRACT}

Cocoa in grain is a tropical fruit considered as a heritage in Ecuador and constitutes $60 \%$ of the premium quality in chocolates worldwide, has sensory characteristics unmatched by other producing countries. Manual drying and selection activities contain many occupational hazards, affecting the safety and well-being of the people involved in these tasks, with the ergonomic risk of an intolerable level requiring immediate intervention. This research provides safe automated mechanisms for operations through internationally validated methodologies, without affecting the sensory characteristics.

Keywords: Sensory characteristics; Occupational hazards; Safety in machinery.

\section{INTRODUCCIÓN}

La cultura del cacao (Theobroma cacao $L$ ) en el Ecuador es ancestral y hereditario, por su historia se sabe que a la llegada de los españoles en la costa del Pacífico, ya se observaban grandes árboles de cacao que demostraban el conocimiento y la utilización de esta especie en la región costera (Paredes, 2009).

Las principales características sensoriales del cacao ecuatoriano es su aroma y sabor, lo que ha hecho distinguirse de los países productores de esta fruta, las exportaciones en el año 2013 fueron de 200.000 toneladas aproximadamente, el $65 \%$ representa el cacao fino de aroma que aporta el Ecuador a nivel mundial. En el año 2011 el Ecuador recibió el premio como el "mejor cacao por su calidad oral" y "mejor grano de cacao por región geográfica” en el Salón du Chocolat en París. (Guerrero, 2013).

Los procesos de secado y selección de cacao en grano son tareas manuales realizados por las personas y están expuestos a diversos riesgos laborales, el no implementar controles operacionales para eliminar o minimizar los riesgos hace que se expongan a accidentes y enfermedades ocupacionales también existen cuantiosas pérdidas económicas, demandas legales, afectación a la imagen empresarial, cierre temporal o permanente de las operaciones el cual tendría un gran impacto económico en miles de ecuatorianos que laboran en este importante sector agrícola e industrial (Bermúdez, 2012).

Según datos de la Organización Internacional de Trabajo, el número de accidentes y enfermedades relacionados con el trabajo, anualmente cobran más de 2 millones de vidas, parece estar aumentando debido a la rápida industrialización. El Institutito Ecuatoriano de Seguridad Social indica que en el 2016 se reportaron 22.861 siniestros laborales, de los cuales $22.179(97,01 \%)$ corresponden a avisos de accidentes de trabajo y $682(2,99 \%)$

1 Ingeniero Químico, MSc en Sistemas Integrados de Gestión, Doctorando en Ingeniería Industrial Universidad Mayor San Marcos, Auditor ISO 9001, 14001, 22001 y OHSAS 18001, más de 12 años de experiencia en empresas nacionales y multinacionales en producción, seguridad, salud y medio ambiente, Ecuador. sergio.nunez@nestle.com.ec

2 Ingeniero de Mantenimiento, Magíster en Educación Superior, Doctorado en Ingeniería Industrial Universidad Mayor San Marcos, actualmente Coordinador de Estación de bombeo de la Estación Corazón, Representante de la dirección en las normas ISO 14001-2004 de la Estación Corazón, encargado de la repotenciación del Poliducto Esmeraldas-Quito, Ecuador. ronal.moscoso@ eppetroecuador.ec

3 Ingeniero Industrial, Diplomado de Ingeniería del Fuego, MSc en Prevención de Riesgo Laboral, Doctorado en Ingeniería Industrial Universidad Mayor San Marcos, Instructor NFPA 1041, más de 14 años de experiencia en sector petrolero en seguridad industrial, salud ocupacional y protección al medio ambiente, Ecuador. alfonso.rivas@eppetroecuador.ec 
corresponden a avisos de enfermedades profesionales.

Los procesos de secado y selección del cacao en grano al realizarlos de manera manual el riesgo ergonómico es el que está presente y mayormente afecta a corto y mediano plazo (Tortosa, García y Ferreras, 1999), señalan que la ergonomía es la disciplina que se encarga del diseño de lugares de trabajo y busca la optimización de los tres elementos del sistema (humano-máquina-ambiente), para lo cual elabora métodos de la persona, técnica y organización.

Actualmente los productores y comercializadores de cacao deberán adoptar medidas para la prevención y minimización de riesgos por lo que se tiene que implementar mecanismos que sean seguros, está estipulado en el Reglamento de Seguridad y Salud de los Trabajadores y Mejoramiento del Medio Ambiente de Trabajo (Decreto Ejecutivo 2393), vigente en Ecuador desde el 1986, teniendo como objetivo la prevención, disminución o eliminación de los riesgos del trabajo y el mejoramiento del medio ambiente de trabajo.

La automatización es una tecnología sólidamente establecida en los sectores manufactureros, donde aspectos como la higiene, la reducción de los costos en su producción, el aumento de la productividad, y minimización de riesgos laborales entre otros, pueden verse beneficiados los empresarios y la sociedad que invierten en este tipo de tecnologías (Ponsa y Granollers, 2013). El Ecuador desde 1995 empieza a trabajar con sistemas mecanizados, siendo las empresas trasnacionales las pioneras en aprovechar de todos sus beneficios, el sector agroindustrial ha tenido retrasos por múltiples factores como el precio de la materia prima, insumos y lo complicado para acceder a tecnología de bajo costo o la realización de equipos por mano de obra nacional o el no disponer del conocimiento apropiado para innovar y desarrollar.

\section{METODOLOGÍA}

El diseño de la investigación es descriptivo y experimental proporcionando un perfil detallado de un evento, condición o situación. El tipo de muestreo fue aleatorio no probabilístico, ya que la muestra utilizada en la investigación fueron lotes de cacao provenientes de diversos cantones de la provincia del Guayas y Los Ríos.

\subsection{Sistema automatizado para el secado del ca- cao en grano}

Prueba 1.- Para el diseño del sistema automatizado, primero se debe estudiar el tipo de combustible como el Diésel o Gas Licuado de Petróleo que se puede usar para el intercambiador de energía, se debe usar fórmulas que se muestra a continuación para determinar la eficiencia de cada combustible:

\section{Determinación del consumo de energía}

(1) $\frac{(100-\text { Humedad final del secado) }}{(100-\text { Humedad inicial del cacao })} \times \frac{\text { Peso de la muestra (Ton) }}{\text { Tiempo }}$

\section{Determinación del consumo de combustible}

(2) Volumen $(\mathrm{Vol})=\frac{\pi}{4} D 2^{*} \mathrm{~h} / \mathrm{T}$

$\mathrm{D}=$ Diámetro en centímetros $(\mathrm{cm}) ; \mathrm{h}=$ Altura en centímetros $(\mathrm{cm}) ; \mathrm{T}=$ tiempo en horas $(\mathrm{h})$

$\pi=3,1415$

\section{Determinación del flujo calorífico}

(3) $\frac{\text { flujo calorífico }\left(\frac{\mathrm{kcal}}{\mathrm{kg}}\right)}{\text { Volumen }(\mathrm{kg}) * \text { Tiempo }(\mathrm{h})}=721,51\left(\frac{\mathrm{kcal}}{\mathrm{h}}\right)$

\section{Determinación del caudal de aire}

(4) Caudal $=\frac{\text { consumo enegético }\left(\frac{k c a l}{h}\right)}{(\Gamma T \times 17)}$

$\Delta T=$ es la diferencia entre la temperatura del aire secado con la temperatura del ambiente

$17=$ es la Constante de Foster

\section{Determinación de la potencia}

(5) $P=V \times A$

$P=$ Potencia eléctrica $(k W): V=$ voltaje (voltios): $A=$ corriente eléctrica (amperios)

Determinación de la eficiencia del secador

(6) $Q=m^{*} c p^{*} \Delta T$

$Q=$ Eficiencia del secador: $m=$ masa: $c p=$ calor especifico

$\Delta T=$ es la diferencia entre la temperatura del aire secado con la temperatura del ambiente

Prueba 2.- Una vez determinada el tipo de combustible a utilizar, se debe analizar e investigar el tipo de diseño a utilizar o en muchos casos una combinación de ellos, los secadores que se 
muestran que se mencionan a continuación son los que hay en mercado para el secado del grano tipo alimenticio:

a) Secador de bandejas. Está conformado por:

- Persianas ajustables

- Bandejas

- Entrada de aire

- Calentador

- Ventilador

- Salida de aire

b) Secador continúo de túnel. Está conformado por:

- Alimentación granular

- Ventilador

- Salida de aire

- Entrada de aire

- Flujo de aire

- Calentadores de vapor de agua

- Transportador de malla

c) Secador de tambor. Está conformado por:

- Campana para vapor

- Alimentador

- Cuchillas

- Tambor con vapor

- Transportador

d) Secador rotatorio. Está conformado por:

- Entrada de aire

- Salida de aire húmedo

- Vapor condensado

- Vapor

- Descarga de sólidos secos

- Alimentador

Prueba 3.- Validar una metodología de determinación de humedad más rápido que el método tradicional (método estufa) que demora 5 horas a un método propuesto (método termobalanza) que los resultados se obtiene en 4 minutos.
2.2 Diseñar un sistema automatizado para la selección del cacao después de haberse secado

Prueba 4.- Investigar si la maquina provista de un equipo con vibración y varias bandejas de diversos diámetros, pueden separar por gravedad el cacao en grano de impurezas y cualquier otro material extraño que pueda afectar a su calidad.

2.3 Determinar las características sensoriales del cacao antes y después de haber secado y seleccionado por medio del sistema automatizado diseñado

Prueba 5.- Realizar pruebas sensoriales con panelistas calificados para determinar los siguientes parámetros con escala del 0 a 10 del: Tostado, Amargo, Quemado, Chocolate, Frutal y Floral, estas pruebas determinan si el secado y seleccionado por medio de los procesos automatizados no han afectado las características sensoriales del cacao. En la Figura 1 se muestra la escala sensorial del cacao en el secado.

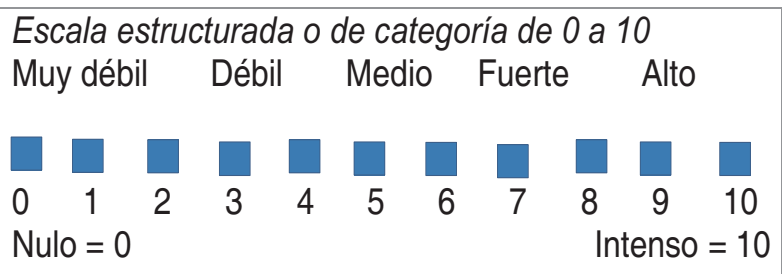

Figura 1. Escala sensorial

Fuente: Elaboración propia

2.4 Evaluar los riesgos laborales asociados a los procesos de secado y selección de cacao de manera manual y automatizada

Prueba 6.- Mediante la metodología Análisis Modo de Falla (AMFE) para identificar peligros y evaluar los riesgos laborales en los procesos de secado y selección, se minimizan en la automatización versus la manera manual, se calcula usando la siguiente formula:

(7) $\mathrm{NPRb}=\mathrm{S} x \mathrm{OxP}$

NPRb =Número de Prioridad de Riesgo Base: $\mathrm{S}=$ Severidad: $\mathrm{O}=$ Ocurrencia: $\mathrm{P}=$ probabilidad

AMFE determina 4 niveles: Bajo, Medio, Alto y Crítico

Bajo: Riesgos laborales controlados

Medio: Riesgos laborales controlados pero requiere supervisión y controles operacionales

Alto: Perjudicial para la salud y que requiere intervención inmediata 
Crítico: Se debe parar las operaciones y reanudar una vez que sean seguras

\section{RESULTADOS}

\subsection{Resultado de la prueba 1}

Se determinó mediante los cálculos el diésel tiene aprovechamiento energético del $86 \%$ y el GLP del $97 \%$.

\subsection{Resultado de la prueba 2}

El tipo de secadora idónea es una de forma circular que posea un sistema de brazos móviles a 1.2 RPM y trabajar a temperatura de $53 \pm 2^{\circ} \mathrm{C}$, no más porque se quema y se forma impurezas y no menor porque el tiempo de secado es más lento, a continuación se muestra el listado de cada uno de los componentes.

\section{Cama}

$1 \mathrm{~A}=$ Plataforma $\mathrm{y}$ bases

$1 \mathrm{~B}=$ Base central de eje

$1 \mathrm{C}=$ Compuerta de descarga

$1 \mathrm{D}=$ Recubrimiento interno de descarga

$1 \mathrm{E}=$ Tolva de transito

\section{Caja de arrastre}

$2 \mathrm{~F}=$ Sujetadores $\mathrm{y}$ bases de cinta

\section{Agitador}

$3 \mathrm{G}=$ Brazos

$3 \mathrm{H}=$ Dispositivo de ascenso y descenso para sistema de descarga

$3 \mathrm{I}=$ Accionamiento del dispositivo de ascenso y descenso

\section{Cercha}

$4 \mathrm{~J}=$ Estructura y bases

\section{Bandeja de secado}

$5 \mathrm{~K}=$ Tina

\section{Compuerta}

$6 \mathrm{M}=$ Compuerta de limpieza

\section{Campana}

$7 \mathrm{~L}=$ Bases para campana

\section{Puerta de descarga}

$8 \mathrm{~N}=$ Puerta manual de descarga

\section{Sistema de transmisión y potencia}

90 = Bases control tracción

$9 \mathrm{P}=$ Piñones

$9 Q=$ Poleas $y$ bandas

$9 \mathrm{R}=$ Ejes

$9 S=$ Cuña

$9 \mathrm{~T}=$ Prisionero

$9 \mathrm{U}=$ Rodamientos

$9 \mathrm{~V}=$ Motor

\section{Turbina}

\section{Quemador}

\section{Base de hormigón}

En la Figura 2 se muestra cada una de las partes detalladas de las maquinas circular y su ubicación respectiva. La vista es desde la parte superior.

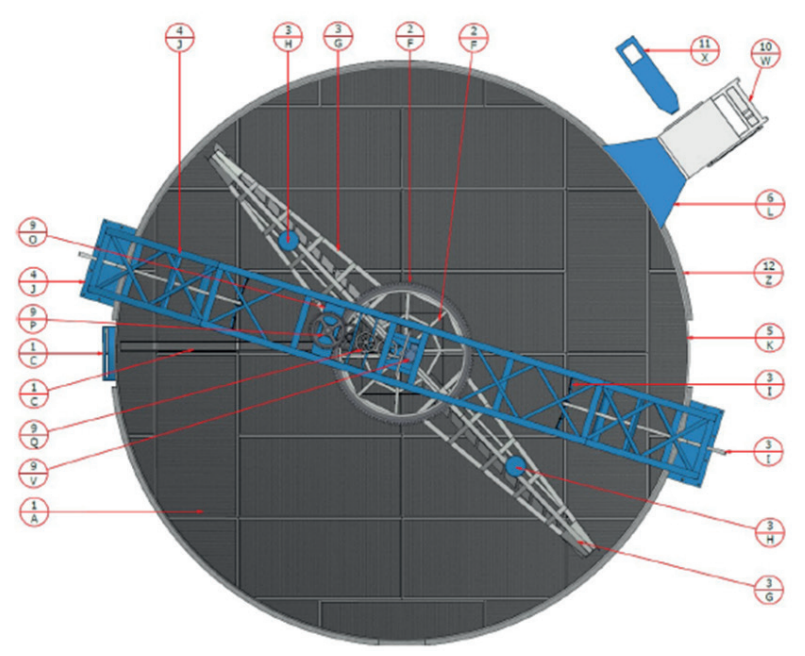

Figura 2. Partes de la secadora circular Fuente: Elaboración propia

En la Figura 3 es una vista general creada en tres dimensiones "3D" de la secadora circular. 


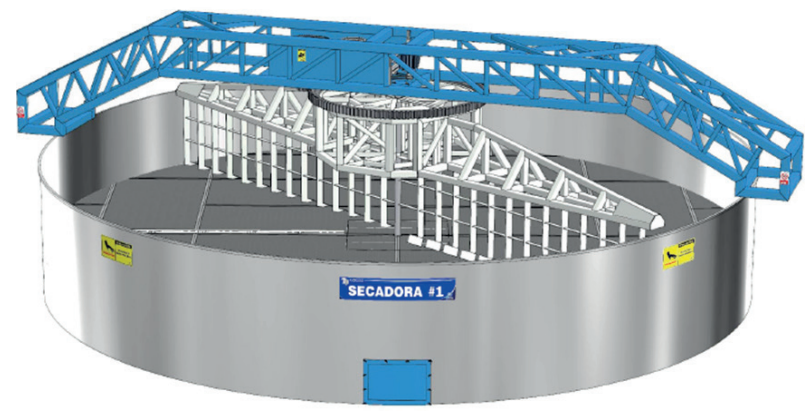

Figura 3. Secadora circular en 3D Fuente: Elaboración propia

\subsection{Resultado de la prueba 3}

Para la validación de la eficiencia en la lectura de la humedad se seleccionó un total de 60 muestras en una población de 100 lotes, fueron secadas de manera manual y divididas en 2 lotes de 100 kilogramos cada uno, el primer lote se determinó con el método estufa (método tradicional) y el segundo lote se determinó mediante el método de la termobalanza (método propuesto), se usó 5 gramos de muestra triturado a un $\mathrm{T}=105^{\circ} \mathrm{C}$ por 4 minutos, existe un nivel de confianza del $99,4 \%$ del método propuesto versus el tradicional.

\subsection{Resultado de la prueba 4}

La máquina seleccionadora está equipada con 3 bandejas:

a. Primera bandeja: Los orificios tienen forma circular que cubre toda la bandeja y un diámetro de $15 \mathrm{~mm}$ (milímetro) con la finalidad de pasar el cacao en grano seleccionado y detener los granos o cualquier otro material extraño con un diámetro superior. La separación de un orificio y otro es de $10 \mathrm{~mm}$ y la cantidad de orificios en la bandeja depende del tamaño de la misma.

b. Segunda bandeja: Los orificios están diseñados en forma oblonga ya no circulares como en la primera bandeja y con las medidas de $13 \mathrm{~mm}$ de ancho y de largo $30 \mathrm{~mm}$ con la finalidad de retener el cacao en grano y dejar pasar cuerpos extraños con un tamaño inferior al diámetro diseñado

En esta segunda fase como está en vibración y la mesa inclinada de 9 a 12 grados el cacao pasa por gravedad.

Recordemos que el peso de un grano de cacao es de 1 a 1.3 gramos y el peso va depender de la variedad del cacao que se está seleccionado y todo cacao con peso inferior al mencionado pasara a la tercera bandeja.

c. Tercera bandeja: En esta bandeja no contiene orificios, en esta bandeja se acumula todos los cuerpos extraños que fueron separados de la segunda bandeja como son las impurezas e inclusive el material particulado o también denominado polvillo de cacao.

\subsection{Resultado de la prueba 5}

Las características sensoriales del cacao se deben primero secar hasta una humedad del $7 \%$ y tomar 500 gramos del lote y hacer un licor de cacao pasando las fases de: Tostado-Triturado-Tamizado-Descascarado-Molienda (Molino IKA M20 o Molino Restch PM100).

Se determinó que secando el cacao en secadoras circulares a $53 \pm 2^{\circ} \mathrm{C}$ y movimientos de 1.2 RPM y seleccionadores por bandejas, el cacao no pierde sus características sensoriales en las 40 muestras de licor que se realizó, no hubo presencia del sabor a quemado, ni tostado. Si el proceso de secado es violento, no se logra un secado uniforme y se interrumpe la hidrólisis enzimática de las antocianinas generando almendras púrpuras que le confieren un sabor astringente, a la vez se endurece rápidamente la testa o cascarilla la cual una vez seca impide la salida o difusión de los ácidos volátiles que se concentran en la almendra generando almendras ácidas.

\subsection{Resultado de la prueba 6}

La metodología Análisis Modo de Falla (AMFE), valora fallas potenciales en el diseño y en la operación previniendo su aparición, cuantificando los efectos de posibles fallas. AMFE determinó que las operaciones se secado y selección del cacao de manera manual son riegos Altos y Críticos que requieren una intervención inmediata, el AMFE de los sistemas automatizados son de riegos Bajos mostrando los procesos más seguros para secar y seleccionar el cacao.

\section{DISCUSIÓN}

El automatizar los procesos de secado y selección del cacao ecuatoriano en las condiciones mencionadas en este proyecto de investigación, van conservar las características sensoriales y minimizar los riesgos laborales, muchas investigaciones realizadas en procesos de secado logran acelerar el proceso pero no se preocupan de los riesgos laborales o miden las características sensoriales, 
haciendo que las investigaciones trabajen por separadas y no de una manera integrada.

\section{CONCLUSIONES}

Dentro de los procesos realizados se concluyó que para hacer un secado y selección del cacao en grano que conserve las características sensoriales se debe usar una temperatura de $53 \pm 2^{\circ} \mathrm{C}$ y $1.2 \mathrm{RPM}$ hasta llegar a una humedad final del cacao al $7 \%$, el diseño y construcción de los de los equipos deben cumplir con normas y estándares de seguridad industrial para la prevención de accidentes y enfermedades laborales.

\section{REFERENCIAS BIBLIOGRÁFICAS}

[1] Bermúdez, F. (2012). Análisis de la seguridad y salud ocupacional en el Ecuador. Ecuador: Editorial Océano.
[2] Guerrero, H. (2013). El Cacao ecuatoriano su historia empezó antes del siglo XV. Líderes, 1(1), 5-8.

[3] Paredes, N. (2009). Manual de cultivo de cacao. Ecuador: Activa.

[4] Ponsa, P., y Granollers, A. (2013). Diseño y automatización industrial. España: Ediciones de la Universidad Politécnica de Catalunya, SL.

[5] Pontiles, B. (2013). Ingeniería en secadores. México: Editorial Mundo Libros

[6] Tortosa, L., García, C., y Ferreras, A. (1999). Ergonomía y discapacidad. Valencia: Instituto de Biomecánica de Valencia (IBV). 